\title{
Comparison of Effectiveness of Pain Management during Manual Vacuum Aspiration Using Single-Agent Analgesic and Combination: A Randomized Double-Blind Controlled Trial
}

\author{
A. Natalia' ${ }^{1,2}$, H. Galadanci ${ }^{1,2}$, S. A. Ibrahim ${ }^{1,2}$, Z. Mohammad ${ }^{1,2}$ \\ ${ }^{1}$ Bayero University, Kano, Nigeria \\ ${ }^{2}$ Department of Obstetrics and Gynaecology, Aminu Kano Teaching Hospital, Kano, Nigeria \\ Email: "drnataliarmu@yahoo.com
}

Received 7 April 2015; accepted 4 May 2015; published 6 May 2015

Copyright (C) 2015 by authors and Scientific Research Publishing Inc. This work is licensed under the Creative Commons Attribution International License (CC BY). http://creativecommons.org/licenses/by/4.0/

\section{(c) (i) Open Access}

\begin{abstract}
Of all the methods available for the management of first trimester miscarriages, manual vacuum aspiration is the safest, cheapest and fastest. However, pain caused by manipulation of cervix and uterine suction makes it uncomfortable. We compared the clinical effectiveness and adverse effects of combination of non-selective COX inhibitor (Diclofenac Sodium 50 mg) and opioid (Pentazocine $60 \mathrm{mg}$ ) to commonly used opiod only (Pentazocine $60 \mathrm{mg}$ ) in daily practice of pain management for the treatment of incomplete abortion with manual vacuum aspiration. This was a randomized double-blind controlled trial conducted in Gynaecological Emergency clinic of Aminu Kano Teaching Hospital Kano, Nigeria. Comparison of the level of pain experienced during the procedure revealed statistically significant difference in the level of pain reported among the groups (P-value 0.03). Comparison of severity of pain perception and patients' satisfaction was found to be negatively related $(P<\mathbf{0 . 0 0 0 0 0 1})$. The present study shows that the use of combined analgesia compared to single agent analgesia during MVA is more, safe, significantly reduced pain and improved patient satisfaction during the procedure.
\end{abstract}

\section{Keywords}

Analgesia, Manual Vacuum Aspiration, Miscarriage, Pain, Pain Management

${ }^{*}$ Corresponding author.

How to cite this paper: Natalia, A., et al. (2015) Comparison of Effectiveness of Pain Management during Manual Vacuum Aspiration Using Single-Agent Analgesic and Combination: A Randomized Double-Blind Controlled Trial. Open Journal of Obstetrics and Gynecology, 5, 244-250. http://dx.doi.org/10.4236/ojog.2015.55036 


\section{Introduction}

Early pregnancy failure occurs in 14\% - 19\% of recognized pregnancies [1]. Incomplete abortion is one of the main causes of maternal mortality and morbidity in the developing countries, as result of haemorrhage and infections. The World Health Organization has provided an estimate that $13 \%$ of pregnancy related deaths globally are due to complications of abortion [2]. Studies from Nigeria has shown that abortion related deaths accounted for $9.6 \%-21.1 \%$ of all maternal deaths, with most of them due to illegally induced abortion, which is the third leading cause of maternal mortality [2].

Of all the methods available for the management of first trimester miscarriages (sharp curettage, electrical vacuum aspiration and manual vacuum aspiration) manual vacuum aspiration is the cheapest, fastest method and associated with fewer incidences of serious complications [3]. However, the main disadvantage of MVA is the pain caused by manipulation of the cervix and uterine suction. It is documented that $97 \%$ of women report moderate to severe intensity pain during and immediately following an abortion [4].

One of the most important aspects in the treating incomplete abortion is the sufficient management of pain during the process of manual vacuum aspiration, with minimal risk to the patient's health and maximum satisfaction. There are varieties of methods used for pain management during surgical miscarriage, which include non-pharmacological method called "verbal anaesthesia", local anaesthesia (paracervical block), opioid and nonsteroidal analgesics, although alone these methods do not provide significant pain control [5] [6]. However, combination of local infiltration of the cervix with Diclofenac and paracervical block; paracervical block with intravenous sedation using Fentanyl and Medozalam documented better pain relief during and after MVA without improved significant patient satisfaction [6]. Recommendations by WHO on safe abortion, as well as by the Royal College of Obstetricians and Gynaecologists favor local/intravenous sedation over general anaesthesia [6]. However, these recommendations were based on data from 1970s to 1980s.

The choice of the method of pain control is guided by doctor's preference, drug availability, financial considerations, individual preference, history of drugs allergy and by institutional consensus. In developing countries, the ideal analgesia should be cheap, easily administered and required minimal resuscitation equipment [7] [8].

In Nigeria including our center, the safest, cheapest and commonly used drugs are Pentazocine and Diclofenac as single agents. The effectiveness of this combination however was not evaluated in a randomized controlled trial (to the best of the author's knowledge based on the available published studies in both electronic \{pubmed, medline\} and written materials sources). Therefore, this study set out to compare the effectiveness of pain management during MVA using single agent (Pentazocine) analgesic and combination (Pentazocine and Diclofenac) in a Randomized double-blind controlled trial.

\section{Materials and Methods}

This study was conducted at Aminu Kano Teaching Hospital (AKTH), which was established in 1988 as the teaching hospital for Bayero University Medical School, located in Kano city, the capital of the Northwestern Nigerian state of Kano. Kano is $99.99 \%$ populated by Africans. The gynaecological emergency clinic is located in a very accessible part of the hospital within the gynaecological ward. It has an average of twenty patients attending in a day out of which $2-3$ require MVA.

Women attending the gynaecological emergencyout-patient clinic of AKTH, Kano diagnosed by clinical evaluation (last menstrual period, earlier ultrasound prior to miscarriage and uterine size) and ultrasound findings showing incomplete abortion, missed abortion or anembryonic pregnancies respectively and those that give consent were included in this study. Patients who had history of allergy to Diclofenac Sodium and Pentazocine and those that did not consent to participate in the study were excluded. This was a randomized double-blind controlled trial. Sample size calculation was performed using formula: $n=\frac{\left(Z_{\alpha}+Z_{\beta}\right)^{2} S^{2}}{d^{2}}$ and assuming a difference in mean pain score among groups of 1.5 and using a standard deviation of $2.8^{9} ; Z_{\alpha}$ - standard normal deviate $=1.96 ; Z_{\beta}$ power $=95 \%=1.64 ; S$ —-standard deviation $=2.8 ; d$-mean difference in pain score $=1.5$.

Forty five women were required in each group to achieve a power of $95 \%$ and type 1 error of 0.05 . This was taken within two months from September 1 and October $30^{\text {th }}$ 2011. Simple Random sampling was used in this study to recruit subjects to compare two groups of pain management protocols during MVA: group I was given Diclofenac and Pentazocine and group II was given Pentazocine only. Patients were assigned to the two groups 
using simple randomization. Allocation was done by balloting. Ninety opaque envelopes containing pieces of paper designated as A-for group one and B-for group two were prepared by a research assistant. In all 45 envelopes were allocated randomly to each group. An envelope was given to each consecutive patient who satisfied the inclusion criteria by an assistant different from the above. Two nurses were recruited for the study, one to administer the analgesic agent and other to assess pain and satisfaction to its management. First aid box was provided to manage emergency complications. The procedure was clearly explained in order to identify and respond to the women's needs. Pre-tested questionnaire were used before the procedure. Diclofenac and Pentazocine were used intramuscularly at standard dose of $1 \mathrm{mg} / \mathrm{kg}$ body weight. A latency period of at least 10 - 20 minutes was given before the procedure. The manual vacuum evacuation was performed according to standard clinical protocol. Each patient was placed in Lithotomy position. Perineum was cleaned with antiseptic solution and draped with sterile towels. The bladder was emptied. A Cusco's speculum was introduced into the vagina and anterior lip of the cervix was held with tenaculum to straighten the endocervical canal. An appropriate size Karman cannula was inserted into the uterine cavity and connected to the prepared vacuum syringe and evacuation was carried out by rocking and rotating movements of the cannulae. The procedure was completed when red foam with gritty sensation seen and felt respectively and no more tissue was seen in the cannula. The cannula, tenaculum and speculum were removed after confirming that the bleeding had stopped. The aspirated tissue was examined and sent for histology. The vital signs were monitored quarter hourly until patient was stable for an hour. Pain rating scale (visual analogue scale) [10] was used during the first three minutes after the procedure and patient satisfaction using Likert scale [11] was used before discharge.

The researcher did not know which agent was used on individual patient before the end of the study so also the subject. The questionnaires were analyzed by the researcher at the end of the study.

All data generated was checked and analyzed using computer based EPI Info version 3.4.1 software. Quantitative variables were described using mean and standard deviation. Qualitative variables were presented as percentages. The student t-test was used to analyze for significant differences between means, chi-square and Mann-Whitney U-test were used for assessing the significance of associations between categorical groups. A P-value of $<0.05$ was considered statistically significant.

Information obtained from the study and findings from the data analysis was presented in tabular, graphical and diagrammatical forms using different variables.

Approval for the study was obtained from the ethical committee of AKTH, Kano.

Approval was sought to interview and manage the patients from the gynaecological emergency department. No patient was forced to participate in this study against her wish. Participation was entirely voluntary. An informed consent form was signed after the nature of the study, the aims and objectives and the protocol were clearly explained to patients.

\section{Results}

A total number of 100 patients were enrolled in the study between the September and October 2011. Of these 100 participants, ten were excluded because they decided not to participate after randomization, 45 were enrolled to group one (Diclofenac and Pentazocine) and 45 patients to group two (Pentazocine only).

Table 1 represents the socio-demographic data and revealed medical histories of the two groups. Overall, these groups were very similar in age, parity and mean gestational age on admission.

The admission diagnoses were anembryonic pregnancies 10 (11.2\%), missed miscarriage 13 (14\%), incomplete 57 (63.3\%), inevitable 9 (10\%), and unsafe miscarriage 1 (1.1\%). The information was displayed in Figure 1.

Only 27 (30\%) of patients were not aware of any methods of analgesia, 31 (34.5\%) heard about it from medical personnel and 25 (27.8\%) from patients.

The mean level of pain in the group one was $4.2(\mathrm{SD} \pm 2.14)$ and $5.6(\mathrm{SD} \pm 2.56)$ in group two. Comparison of the level of pain experienced during the procedure revealed statistically significant difference in the level of pain reported among the groups (P-value 0.03). Data was summarized in the Table 2.

Patients satisfaction was better in the first group 37 (82.2\%), compared to group two 30 (66.6\%). Women who received Pentazocine only had higher dissatisfaction 6 (13.4\%) compared to women in group one 3 (6.7\%). A comparison of the level of participant's satisfaction experienced during procedure revealed no statistically significant difference between the groups, as displayed on Table 3.

Majority of patients 61 (85.9\%) experiencing low to moderate pain (0 - 7) were satisfied, while only 6 (31.6\%) 


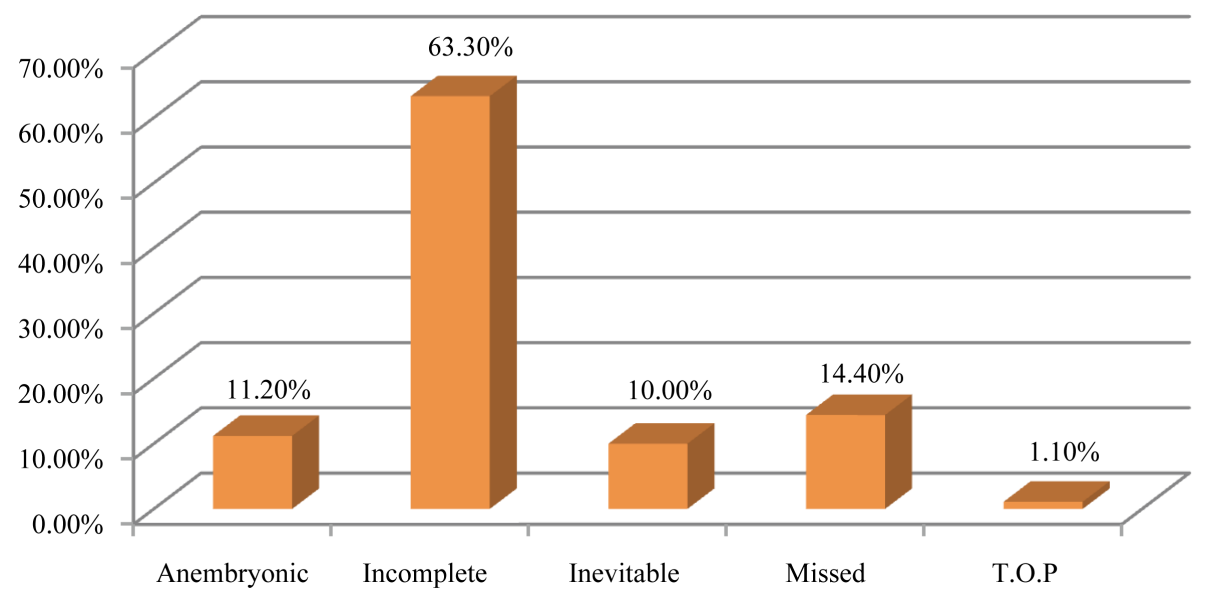

Figure 1. Type of miscarriage. The admission diagnoses were anembryonic pregnancies 10 (11.2\%), missed miscarriage $13(14 \%)$, incomplete 57 (63.3\%), inevitable $9(10 \%)$, and unsafe miscarriage $1(1.1 \%)$.

Table 1. Socio-demographic characteristics of the participants.

\begin{tabular}{|c|c|c|c|}
\hline Variable & $\begin{array}{c}\text { Group I } \\
\text { (Diclofenac and pentazocine) }\end{array}$ & $\begin{array}{c}\text { Group II } \\
\text { (Pentazocine only) }\end{array}$ & P-value \\
\hline Mean maternal age & $27.88(\mathrm{SD} \pm 7.02)$ & $28.77(\mathrm{SD} \pm 7.14)$ & 0.55 \\
\hline Mean parity & $4.3(\mathrm{SD} \pm 3.43)$ & $3.6(\mathrm{SD} \pm 2.58)$ & 0.27 \\
\hline Mean gestational age & $10.0(\mathrm{SD} \pm 2.33)$ & $9.9(\mathrm{SD} \pm 2.10)$ & 0.74 \\
\hline Mean prev Preg loss & $0.95(\mathrm{SD} \pm 1.14)$ & $0.84(\mathrm{SD} \pm 1.04)$ & 0.63 \\
\hline Variable & Group I & Group II & \\
\hline \multicolumn{4}{|l|}{ Religion } \\
\hline Christianity & $2(4.4 \%)$ & $3(6.7 \%)$ & \\
\hline Islam & 43 (95.6\%) & 42 (93.3\%) & \\
\hline Total & $45(100 \%)$ & $45(100 \%)$ & \\
\hline Fisher exact test & P-value $=0.5$ & & \\
\hline \multicolumn{4}{|l|}{ Occupation } \\
\hline Housewife & 39 (86.7\%) & 35 (77.7\%) & \\
\hline Civil servants & $6(13.3 \%)$ & $10(22.3 \%)$ & \\
\hline Total & $45(100 \%)$ & $45(100 \%)$ & \\
\hline
\end{tabular}

$\mathrm{X}^{2}=1.22 ; \mathrm{P}$-value $=0.2$.

Table 2. Level of pain by treatment type.

\begin{tabular}{cccc}
\hline Level of pain & $\begin{array}{c}\text { Group I } \\
\text { (Diclofenac and pentazocine) }\end{array}$ & $\begin{array}{c}\text { Group II } \\
\text { (Pentazocine only) }\end{array}$ & \\
\hline $0-4$ (mild) & $29(64.4 \%)$ & $18(40.0 \%)$ & \\
$5-7$ (moderate) & $13(28.9 \%)$ & $11(24.4 \%)$ & \\
$8-10$ (severe) & $3(6.7 \%)$ & $16(35.6 \%)$ & \\
Total & $45(100 \%)$ & $45(100 \%)$ & \\
Mean level of pain & $4.2(\mathrm{SD} \pm 2.14)$ & $5.68(\mathrm{SD} \pm 2.58)$ & P-value 0.03 \\
\hline
\end{tabular}

$\mathrm{X}^{2}=11.64 ;$ P-value $=0.029$. 
with severe pain (8 - 10) were satisfied with pain management. Only few $2(2.8 \%)$ of the patients despite lower pain perception were still dissatisfied. Comparison of severity of pain perception and patients' satisfaction was found to be negatively related ( $<0.0000012)$. Data was shown in Table 4.

There were no major complications in either group, no procedure was moved to the operation theater or converted to general anaesthesia due to patient discomfort.

Majority of patients in group one 20 (44.4\%) reported no side effects. The most common side effects reported among patients of both groups were dizziness 19 (42.2\%) and 39 (86.6\%) in the first and second groups respectively, followed by nausea and vomiting 6 (13.4\%) and $3(6.7 \%)$. There were significantly more side effects in the group with Pentazocine only.

Significantly higher number of participants in group one 38 (84.4\%) would choose the same analgesia during the next procedure compared to group two 29 (64.4\%). Yet 16 (35.6\%) of patients in group two would prefer general analgesia compared to group one 7 (15.6\%).

\section{Discussion}

First trimester uterine bleeding due to incomplete abortion continues to be one of the main causes of maternal morbidity and contributes $13 \%$ of all maternal deaths globally [2]. World Health Organization has listed Manual Vacuum Evacuation as an effective and safe method of uterine evacuation and hence the technique is being employed increasingly in the developing world under minimal anaesthesia or sedation in the management of incomplete miscarriages [5]. However, pain control is an important and relevant issue in managing incomplete miscarriages using manual vacuum aspiration (MVA).

This study showed a statistically significant difference in pain level between group I (Pentazocine plus Diclofenac) and group II (Pentazocine only) meaning that the use of combine analgesia provided more pain relief compared to the use of single agent analgesia. This differed with the findings of Lopez et al. were they documented that there was no difference between the use of Meperidine (an opioid like Pentazocine) plus Diclofenac and the use of Meperidine alone. This could be explained by the difference in the pharmacology of Pentazocine compared to Meperidine. While Meperidine can increase level of anxiety [9], Pentazocine does the opposite with probable resultant effect of decreasing pain score. In addition the documented association of better one hour post operative pain relief with Diclofenac [6] could have added to the better pain relief in group one compared to group II.

This study showed that participants of both group I and II were satisfied with the two type of treatment given. This might not be unconnected with the age-long preference of injection (injections were used in this study) as

Table 3. Patients satisfaction by treatment type.

\begin{tabular}{ccc}
\hline Level of pain & $\begin{array}{c}\text { Group I } \\
\text { (Diclofenac and pentazocine) }\end{array}$ & $\begin{array}{c}\text { Group II } \\
\text { (Pentazocine only) }\end{array}$ \\
\hline Neither satisfied or dissatisfied & $5(11.1 \%)$ & $9(20.0 \%)$ \\
Dissatisfied & $3(6.7 \%)$ & $6(13.4 \%)$ \\
Satisfied & $37(82.2 \%)$ & $30(66.6 \%)$ \\
Total & $\mathbf{4 5 ( 1 0 0 \% )}$ & $\mathbf{4 5 ( 1 0 0 \% )}$ \\
\hline
\end{tabular}

$\mathrm{X}^{2}=2.87 ;$ P-value $=0.23$.

Table 4. Patients satisfaction by severity of pain.

\begin{tabular}{ccc}
\hline Level of pain & $0-7$ & $8-10$ \\
\hline Neither satisfied or dissatisfied & $8(11.3 \%)$ & $6(31.6 \%)$ \\
Dissatisfied & $2(2.8 \%)$ & $7(36.8 \%)$ \\
Satisfied & $61(85.9 \%)$ & $6(31.6 \%)$ \\
Total & $\mathbf{7 1 ( 1 0 0 \% )}$ & $\mathbf{1 9}(\mathbf{1 0 0} \%)$
\end{tabular}

$\mathrm{X}^{2}=27.27 ;$ P-value $=0.000001$. 
form treatment in this environment [12] not necessarily because of the pain relief experienced by the participants in both groups. As would be expected participants in both groups with lower level of pain score $(0-7)$ expressed more satisfaction than those with higher score $(8$ - 10).

There were no major complications in either of the groups in this study. However, there were more reported side effects among participants in group II compared to group I. Up to $86.6 \%$ with dizziness in group II compared to $42.2 \%$ in group I.

Participants' preference of injections during the next procedure compared to general anesthesia in this study was statistically significant. This could partly due to their satisfaction with pain relief from the treatment given or partly due same reason reported by Elisha [12]. However, a trend was observed between group I and II in preference for general anesthesia during the next procedure of $15.6 \%$ and $35.6 \%$ respectively, this could still be due to the difference in satisfaction with pain relief based on the treatment type rather than simply due to preference for injections.

There was no statistically significant difference among the groups in term of age with mean age of group I being 27.88 ( $\mathrm{SD} \pm 7.02$ ), that of group II being 28.77 ( $\mathrm{SD} \pm 7.14$ ) and $\mathrm{P}=0.55$. The mean age of both groups were similar to what was documented by Omole-Ohonsi et al. in a study conducted at the AKTH among similar population on the sociodemographic characteristics and etiological factors of vaginal discharge in pregnancy [13]. Though, these were slightly lower than the mean age found by Lopez et al. of $29.1 \pm 5.3$ and $30.3 \pm 6.3$ in Meperidine plus Diclofenac and Meperidine groups respectively. The explanation could be due to lower age of marriage among females in Northern Nigeria [14]. The two groups were comparable in term of mean parity $(\mathrm{P}=$ 0.27 ) to themselves and to similar population previously studied [13]. They were also comparable in gestational age $(0.74)$ and pregnancy loss $(P=0.74)$. The groups were similar in distribution by religion $(P=0.5)$, occupation $(\mathrm{P}=0.27)$ and level of education $(\mathrm{P}=0.95)$.

This study found that only $30 \%$ of the participants had no information whatsoever about analgesia for MVA compared to $70 \%$ who had information through various medium which included $34.5 \%$ from health workers, $27.8 \%$ from other patients, $5.5 \%$ radio, $1.1 \%$ seminars and $1.1 \%$ from mosque.

\section{Conclusion}

The present study shows that the use of combined analgesia compared to single agent analgesia during MVA is more, safe, significantly reduced pain and improved patient satisfaction during the procedure.

\section{Recommendations}

1) Introduction of the use of Pentazocine plus Diclofenac can offer an opportunity for effective pain management and improve patient satisfaction in the Gynecological Emergency of AKTH and similar departments across Nigeria.

2) A prospective multi-centered, double blind controlled trial comparing the effectiveness and adverse side effects of the agents used in this study is recommended for stronger conclusion and generalization.

3) Personnel should discard the notion that pain control medication is unnecessary and consider patients choice of analgesia during MVA.

\section{Limitations}

This study did not represent all women of Kano State who experienced early pregnancy loss, especially low income women, receiving free services in the state hospitals.

Higher number of doctors was recruited. It might influence pain perception by his or her approach to MVA procedure and counseling the patient regarding the discomfort they might have.

\section{References}

[1] Palton, V., Harris, L., Weisman, C., Guire, K., Gastleman, L. and Lebovic, D. (2006) Patient Preferences, Satisfaction and Resource Use in Office Vacuation of Early Pregnancy Failure. Journal of Obstetrics and Gynaecology, 101, 103-110.

[2] Nwogu-Ikojo, E.E. and Ezegwui, H.U. (2007) Abortion-Related Mortality in a Tertiary Medical Center in Enugu, Nigeria. Journal of Obstetrics and Gynaecology, 27, 835-837. http://dx.doi.org/10.1080/01443610701718883 
[3] Koontz, S.L. Molina de Perez, O., Kathleen, L. and Foster-Rosoles, A. (2003) Treating Incomplete Abortion in El Salvador: Cost Saving with Manual Vacuum Aspiration. Contraception, 68, 345-351. http://dx.doi.org/10.1016/S0010-7824(03)00162-8

[4] Gweneth, B.L., Nicholas, S.F. and Tod, F. (2009) Impact of Paracervical Block on Postabortion Pain in Patients Undergoing Abortion under General Anaesthesia. Contraception, 80, 78-82.

[5] Gomez, P.I., Gaitan, H., Nova, C. and Paredas, A. (2004) Paracervical Block in Incomplete Abortion Using Manual Vacuum Aspiration: Randomized Controlled Trial. Obstetrics \& Gynecology, 103, 943-951. http://dx.doi.org/10.1097/01.AOG.0000123269.86525.c4

[6] Renner, R.M., Jensen, J.T., Nichols, M.D. and Edelman, A. (2009) Pain Control in First Trimester Surgical Abortion. Cochrane Database of Systematic Reviews, 15, Article ID: CD006712.

[7] Owolabi, O.T. and Moodley, J. (2005) A Randomized Trial of Pain Relief in Termination of Pregnancy in South Africa. Tropical Doctor, 35, 136-139. http://dx.doi.org/10.1258/0049475054620923

[8] Lopez, J.C., Vigil-De Gracia, P., Vega Malek, J.C., Ruiz, E. and Vergara, V. (2007) A Randomized Comparison of Different Methods of Analgesia in Abortion Using Manual Vacuum Aspiration. International Journal of Gynecology Obstetrics, 99, 91-94. http://dx.doi.org/10.1016/j.ijgo.2007.05.023

[9] Schug, S.A. (2010) Cox-2 Inhibitors for the Management of Postoperative Pain. Anästhesiologie, Intensivmedizin, Notfallmedizin, Schmerztherapie, 45, 56-63.

[10] (2009) Pain Measuring Editor’s Notes. Penton Media. www.analgesia/measuringpain.mht

[11] Liket Scale. www.analgesia/Likert scale.mht

[12] Elisha, P.R. (2010) The Politics of Polio in Nigeria. Indiana University Press, Bloomington. www.books.google.com/books

[13] Omole-Ohonsi, A. and Nwokedi, E.E. (2011) Sociodemographic Characteristics and Etiological Factors of Vaginal Discharge in Pregnancy. Jos Journal of Medicine, 5.

[14] Andrew, S.K. (2009) Addressing Child Marriage in Northern Nigeria. www.popcouncil.org/project 